dence of malignancy and the histological finding was in keeping with sarcoidosis (figs 2 and 3).

\section{Questions}

(1) What is the differential diagnosis?
(2) What does the chest radiograph show?

(3) What is the cause of deafness in the left ear?

(4) What are the otolaryngological manifestations of sarcoidosis?

\title{
A difficult psychiatric patient
}

Answers on p 590.

Department of Old

Age Psychiatry,

Maudsley Hospital, Denmark Hill, London SE5 8AZ, UK

Correspondence to: Dr Henderson

Submitted 13 July 1999 Accepted 10 September 1999

\author{
Max J Henderson
}

A 74 year old man was admitted to a psychiatric ward at the request of his community psychiatric nurse. He arrived unaccompanied by either his nurse or his family. The history in the community psychiatric nurse's letter was that the patient's family had been concerned for the past week as he had taken to his bed. They had needed to wash and feed him. He slept a lot. They had alerted the nurse who, having seen the patient at home, arranged for an urgent admission.

From the old medical notes it was clear the patient had a long psychiatric history dating back 40 years. His initial diagnosis was obsessive-compulsive disorder but the majority of his admissions had been for agitated depression. He had taken at least one overdose in the past. There was a documented history of alcohol abuse, but it was not clear if this was still an issue. More recently he had developed idiopathic Parkinson's disease. Cognitive impairment had been noted on his last admission: computed tomography had showed cerebral atrophy and some small infarcts and he had been started on aspirin. His medication which accompanied him on admission also included paroxetine, lithium, and co-beneldopa (Madopar).

Very little history was available from the patient, who needed to be roused from sleep.
He said he felt "terrible" but could not elaborate. $\mathrm{He}$ denied any pain. $\mathrm{He}$ admitted being sleepy. No clear psychotic features were noted but it was questioned whether or not the patient understood.

Examination of the patient's cardiovascular and respiratory systems was unremarkable as was that of the abdomen. Neurological examination revealed normal cranial nerves. Parkinsonian features were clearly demonstrated with rest tremor and cogwheel rigidity worse on the left. The patient could walk but needed assistance and conclusions about his gait were not drawn. Reflexes were normaland symmetrical-plantars were both downgoing.

Initial results showed a normal haemoglobin, a slightly raised white cell count $\left(12.3 \times 10^{9} / 1\right)$, normal urea, creatinine, and electrolytes, normal glucose on BM finger prick testing and normal urine dipstick.

\section{Questions}

(1) What is the diagnosis in this patient and what would you also consider?

(2) What particular risk factors for this condition were present in this patient?

(3) How is this condition normally managed? 


\section{A rare case of radiculopathy in an elderly man}

Answers on p 591.

Level 5, Belfast City

Hospital, Lisburn

Road, Belfast

BT9 7AB, UK

W K Edrees

B Lee

Correspondence to:

Mr Edrees

(email:

wkedrees@hotmail.com)

Submitted 22 September 1999

Accepted 8 October 1999
W K Edrees, B Lee

An 82 year old man was admitted to a district general hospital with a two week history of pyrexia, low back pain referred down the left leg, and progressive weakness of his left leg. On examination he was pyrexic, $38.5^{\circ} \mathrm{C}$, with signs of lower motor neurone lesion extending from L2 to S1 with no other findings. His haemoglobin concentration was $78 \mathrm{~g} / 1$, white cell count $7.7 \times 10^{9} / 1$, and erythrocyte sedimentation rate $115 \mathrm{~mm} /$ hour.

Computed tomography for the LS spine showed no cord compression and identified a lesion at the left psoas muscle. Subsequently, computed tomography of the abdomen was performed (see fig 1). This was followed by aspiration and a core biopsy under computed

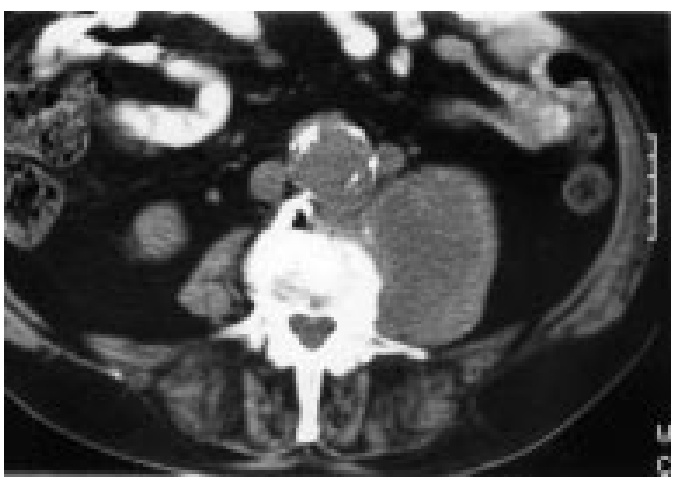

Figure 1 Computed tomogram of the abdomen. tomographic guidance. Bacteriology was negative and cytology non-conclusive. The patient was treated empirically with antibiotics and his symptoms gradually settled down.

Seven weeks later, the patient experienced sudden severe abdominal pain and a large mass was apparent in the left side of the abdomen. Computed tomography with enhancement was performed (see fig 2).

\section{Questions}

(1) Describe the findings in fig 1?

(2) Describe the findings in fig 2?

(3) How the patient was managed and what was the diagnosis?

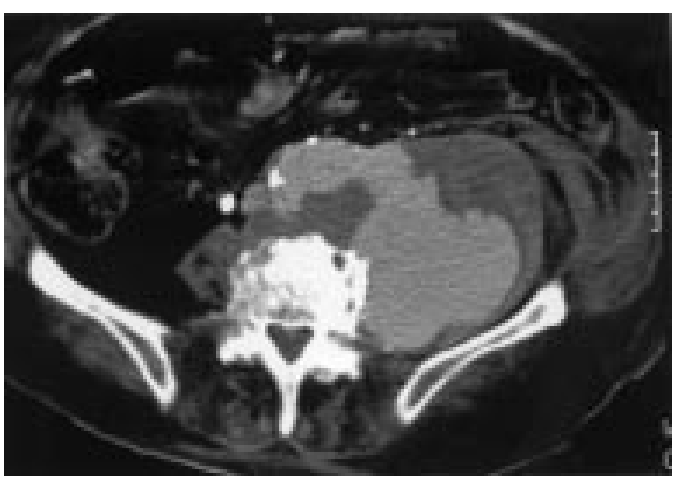

Figure 2 Computed tomogram with enhancement.

\section{How can fruits and sugar induce headache and hypoglycaemia?}

Victor Y Kopyev, Eugene Lozner

A 47 year old white woman, while she was being evaluated for atypical chest pain, mentioned that she had had a life long history of a "sugar intolerance". On further questioning she described her symptoms as a severe headache, sweating, nausea, vomiting, abdominal pain, and fainting occurring shortly after she ate something sweet. She had had these symptoms since infancy from the time she was weaned. She developed an aversion to sweets and followed a diet, which consisted mainly of meat and dairy products. She had a brother who had the same problem. Ten to 20 years ago she was given a "glucose tolerance test" by her family physician who gave her a glass of sweetened orange juice. She developed a severe attack of her usual symptoms, shaking, and perspiration and she almost lost consciousness. Hypoglycaemia was documented by her physician. Fortunately, she recovered spontaneously. At that time her doctor recommended that she continue with her diet. She remained well and was able to stay almost symptom-free by carefully selecting what she ate. Her review of systems and physical examination were also remarkable for excellent dental health.

\section{Questions}

(1) What diagnosis would account for her "sugar intolerance" and what diagnostic test would you recommend?

(2) How would you treat hypoglycaemia in such patients?

(3) What needs to be done for the patient? 